BULL. AUSTRAL. MATH. SOC.

\title{
LATTICE COVERINGS AND THE DIAGONAL GROUP
}

\author{
G. RAMHARTER
}

Let $M$ be any bounded set in $n$-dimensional Euclidean space. Then almost all n-dimensional lattices $L$ with determinant 1 have the following property: There exists a diagonal transformation $D$ with determinant 1 (depending on $L$ ) such that $L$ does not cover space with $D M$. Moreover, if $M$ has non-empty interior, the exceptional (null-) set contains at least enumerably many diagonally nonequivalent lattices.

1. Let $L$ denote the space of lattices in $n$-dimensional Euclidean space $\mathbb{R}^{n}$ with determinant 1 , equipped with the usual measure and topology (see [11] Section 17, Section 19), and let $D$ be the group of nonsingular diagonal $n \times n$-matrices. The main purpose of this note is to prove the following result.

PROPOSITION. Let $M$ be a bounded set however large in $\mathbb{R}^{n}$. Then all lattices $L \in L$ except those from a null-set in $L$ (in the sense of the measure introduced) have the following property: $L$ can be made a noncovering lattice for $D M$ by applying a suitable diagonal transformation $D \in D$ (depending on $L$ ) with $|\operatorname{det} D|=1$.

We will obtain this as a corollary of the Theorem to be stated below.

Received 28 May 1986.

Copyright Clearance Centre, Inc. Serial-fee code: 0004-9727/87 $\$ A 2.00+0.00$. 
Also it will become clear from (l) that our assertion is certainly not trivial at least for sets $M$ with non-empty interior. The present result can be regarded as a metrical contribution to the following general inhomogeneous problem of the "first type" (in Bambah's terminology [1] p.120; see also [7] p.208, and [11] p.407): Given some class $M$ of (measurable) sets in $\mathbb{I R}^{n}$ and a subgroup $G$ of the general linear group on $\mathbb{R}^{n}$, determine the infimum $\delta(M)$ of all positive numbers $\delta$ with the property that, for any $M \in M$ with $\operatorname{vol}(M) \leq \delta$ and any lattice $L \in L$, there exists a transformation $G \in G$ such that the Minkowski sum $G M+L$ does not cover $\mathbb{R}^{n}$. Taking in particular (in view of our present objectivel the diagonal group $D$, we reformulate this by introducing the function

$$
\delta_{M}(L)=\sup \{\operatorname{vol}(D M) \mid D \in D, L \text { not a covering lattice for } D M\}
$$

For bounded $M$ this quantity is positive, possibly infinite (Note that $I^{n}$ is not covered by $D M+L$ if and only if $L$ is strictly admissible for some translate of $D M$ ). Then obviously

$$
\delta(M)=\inf \left\{\delta_{M}(L) \mid M \in M, L \in L\right\} .
$$

This should be compared with the corresponding covering problem of the second type, involving the quantity

$$
\Theta_{M}(L)=\inf \{v o Z(D M) \mid D \in D, L \text { a covering lattice for } D M\} \text {. }
$$

D.B. Sawyer [15] proved that for arbitrary lattice $L \in L$ one has

$$
\sup _{K} \theta_{K}(L)=n^{n} / n !
$$

where the sup is taken over all n-dimensional convex bodies $K$, not necessarily 0 -symetric (Indeed, by the affine invariance of this class of sets, this need only be proved for the integer lattice $\mathbb{Z}^{n}$ ).

We remark that there is an analogous packing problem in connection with the diagonal group which was suggested by Mordell in 1936 and has attracted much interest since (see for example [11] Section 24, [7] p.191 f.). It is concerned with the function 


$$
\rho_{K}(L)=\sup \{v o l(D K) \mid D \in D, L \text { a packing lattice for } D K\}
$$

If $K$ is restricted to the class $K$ of 0 -symmetrical bounded convex bodies in $\mathbb{R}^{n}$, then trivially $\rho_{K}(L) \leq 1$, by Minkowski's theorem. The question is to decide whether the values of this function are bounded away from 0 when $K$ and $L$ are varying over $K$ and $L$. Rogers [14] confirmed this by finding explicit positive lower bounds in all dimensions. These estimates can be improved when further conditions are imposed on the bodies considered. This was done by Hlawka [10] and Davenport for the classes of parallelepipeds $D B \quad(D \in D, B$ the unit cube) and ellipsoids DS ( $S$ the unit sphere), respectively.

A conjecture by Gruber claiming that $\rho_{B}(L)$ takes the value 1 (which is the greatest possible value) at almost all $L \in L$ is still open for $n \geq 3$ (for metrical and topological results in this direction see $[8,12])$.

2. Here we obtain an affirmative answer to the inhomogeneous version of this conjecture. The following Theorem clearly implies the above Proposition.

THEOREM. Let $M$ be cony bounded (measurable) set in $\mathbb{I R}^{n}$ with nonempty interior and let $L^{\prime}$ denote the set of lattices $L \in L$ with $\delta_{M}(L)<\infty$. Then

(1) L' contains an enumerable set of diagonally inequivalent lattices; moreover, $\delta(M)=\inf \left\{\delta_{M}(L) \mid L \in L\right\}$ is a finite positive number.

$$
\text { L' is a nulz-set in } L \text {. }
$$

Proof. We find it convenient to consider the Minkowski sets ${ }^{B_{p}}:\left(\left|x_{1}\right|^{p}+\ldots+\left|x_{n}\right|^{p}\right)^{1 / p}<1$, that is the (open) unit balls of the $p$-norms $(1 \leq p \leq \infty)$. In particular, letting $p$ tend to $\infty$, we obtain the unit cube $B=B_{\infty}$. By $\Omega_{i}\left(i=1, \ldots, 2^{n}\right)$ we denote the (open) coordinate orthants, listed in any order. We introduce the auxiliary function 
$\alpha_{p}(L)=\max _{i=1, \ldots, 2^{n}}^{\sup \left\{\operatorname{vol}\left(D B_{p} \cap \Omega_{i}\right) \mid D \in D, \quad L \text { admissible for } D B_{p} n \Omega_{i}\right\}}$. We remark that the functions $\alpha_{p}(L)$ and $\delta_{B}(L), B=B_{p}$, can be described in terms of a semiregular continued fraction expansion for twodimensional lattices (see [13]). We collect some basic relations between the above functions. First, it follows immediately from the definition that $\delta_{M}(L)$ is diagonally invariant with respect to both $M$ and $L$ : for any set $M$ and any $L \in L$ we have

$$
\begin{array}{ll}
\delta_{D M}(L)=\delta_{M}(L) & (D \in D), \\
\delta_{M}(D L)=\delta_{M}(L) & (D \in D,|\operatorname{det} D|=1) .
\end{array}
$$

By use of (3a), it is easily proved that for any bounded set $M$ with non-empty interior (as specified in the Theorem) the inequalities

$$
(4 a, b) \quad c \delta_{B}(L) \leq \delta_{M}(L) \leq c^{\prime} \delta_{B}(L)
$$

hold with some positive constants $c, c^{\prime}$ independent of $L$. Here $c$ (respectively $C^{\prime}$ ) may be taken as the ratio $\operatorname{vol}(M) / \operatorname{vol}(D B)$ where $D \in D$ is any diagonal matrix such that a translate of $M$ can be inscribed in $D B$ (respectively $D B$ is contained in some translate of int $M$ ). Next we show that

$(5 a, b)$

$$
n ! n^{-n} \alpha_{1}(L) \leq \delta_{B}(L) \leq 2^{n-1} \alpha_{\infty}(L)
$$

The first inequality $(5 a)$ is easily obtained on comparing the volumes of a (lattice point free) simplex of the form $D T\left(T: x_{1}+\ldots+x_{n}<1\right.$, $\left.x_{1}, \ldots, x_{n}>0\right)$ and a maximal inscribed translate of a parallelepiped of the form $D^{\prime} B\left(D, D^{\prime} \in D\right)$. For the proof of (5b) take any lattice point free parallelepiped of the form $D B+z, z \in R^{n}$. Eventually enlarge it by moving appropriate facets outward until at least one facet contains a lattice point in its relative interior (the volume will not be decreased by this process). By passing to a suitable translate $P^{\prime}$, if necessary, we may assume that the boundary of $P^{\prime}$ contains the origin 0 . Now $P^{\prime}$ is the disjoint union of $i$ ts non-empty intersections $P_{i}\left(2^{n-1}\right.$ in number) with the open orthants, and its intersections with the coordinate planes (which do not contribute to the volume). $P^{\prime}$ 
being lattice point free, the same is true for the subsets $P_{i}$. Now (5b) follows from the fact that these subsets are all of the form $D_{i} B \cap \Omega_{i}$, $D_{i} \in D$.

We proceed to the proof of (1). First we exhibit an enumerable class of lattices $L^{*}$ generated by real number fields for which $\delta_{M^{*}}\left(L^{*}\right)$ is finite. By $(4 b)$ and $(5 b)$ it is enough to show that $\alpha_{\infty}\left(L^{*}\right)$ is finite for these lattices. Let $A$ be an $n \times n$-matrix whose elements $a_{11}, \ldots, a_{1 n}$ form a basis of a totally real number field of degree $n$, the $k$-th column consisting of the conjugates of $a_{1 k}(k=1, \ldots, n)$. Then $L^{*}=A^{*} \mathbb{Z}^{n}, A^{*}=A /|\operatorname{det} A|^{1 / n}$, is in $L$. For the calculation of $\alpha_{\infty}\left(L^{*}\right)$ it suffices to consider, in each orthant $\Omega_{i}$, the (enumerable) system of lattice point free (open) parellelepipeds $P_{i j}$ of the form $D_{j} B \cap \Omega_{i}$ each of whose facets not contained in a coordinate plane has a lattice point in its relative interior. It is known [3-6] that under the above assumptions this system is periodic in the following sense: For each $i=1, \ldots, 2^{n}$, there exists a finite subsystem of parallelepipeds $P_{i 1}, \ldots, P_{i m(i)}$, say, such that any $P_{i j}$ is representable as $D P_{i k}$ with some $k \in\{1, \ldots, m(i)\}, D \in D,|\operatorname{det} D|=1$. Therefore $\alpha_{\infty}\left(L^{*}\right)=\max _{i, k}$ $\left\{\operatorname{vol}\left(P_{i k^{\prime}}\right)\right\}$, but this is clearly finite. Thus we have proved that $\delta_{M}\left(L^{*}\right)$ is finite for any lattice of the type described.

Finally, Hlawka's result [10] ensures the existence of an (explicit) positive lower bound, depending only on the dimension, for the values of $\rho_{B}(L)$. Together with the inequality $(4 a)$ and the trivial estimate $\delta_{B}(L) \geq \rho_{B}(L)$ this implies that $\inf _{L} \delta_{B}(L)$ is positive, which completes the proof of (1).

We turn to the proof of (2). Consider the following conditions for lattices $L \in L$ :

(a) $L \backslash\{\underline{0}\}$ has no points in common with the coordinate planes; 
(b) there exists a sequence of diagonal transformations $D_{i} \in D$ with $\left|\operatorname{det} D_{i}\right|=1(i=1,2, \ldots)$, depending on $L$, such that the lattices $L_{i}=D_{i} L$ have bases $A_{i}$ converging (elementwise) to a nonsingular matrix $A Q$ where $Q$ is an $n \times n$-permutation matrix and $A$ contains an $(n-m) \times m$ rectangular block $(1 \leq m \leq n-1)$ of zeros below the diagonal; (c) the homogeneous minimum $\inf \left\{\left|x_{1} x_{2} \cdots x_{n}\right| \mid \underline{x} \in L \backslash\{\underline{0}\}\right\}$ is equal to 0 . It follows from a result of Birch and Swinnerton-Dyer [2] that (c) implies (b). On the other hand, it is well-known that almost all lattices in $L$ satisfy conditions (a) and (c). Accordingly, for the verification of (2), it will suffice to prove that $\delta_{M}(L)=\infty$ if $L$ has properties (a) and (b). We do this by showing that $\alpha_{1}\left(L_{i}\right)$ tends to $\infty$, as $i \rightarrow \infty$. Let $a_{1}^{(i)}, \ldots, a_{n}^{(i)}$ be the columns of $A=\left(a_{k j}^{(i)}\right)$. After renumbering the coordinate axes, if necessary, we may suppose that $Q$ is the identity matrix. Then, for each pair of indices $k, j, k=m+1, \ldots, n ; j=1, \ldots, m$, the elements $a_{k j}^{(i)}$ tend to 0 , as $i \rightarrow \infty$. Let $E_{i}$ denote the $(n-1)-$ dimensional lattice plane generated by the points $0, a_{1}^{(i)}, \ldots, a_{n-1}^{(i)}$. There is a unique vector $\underline{e}^{(i)}$ orthogonal to $E_{i}$ and normalized by the conditions $\left|\underline{e}^{(i)}\right|=1, d_{i}=\left(\underline{e}^{(i)} \underline{a}_{n}^{(i)}\right)>0$. Let $h(i)$ be the index of the orthant which contains the point $\underline{e}^{(i)}$. Since $\underline{e}^{(i)}$ belongs to the orthogonal complement of the subspace generated by $a_{1}^{(i)}, \ldots, a_{m}^{(i)}$, the components $e_{j}^{(i)}(j=1, \ldots, m)$ tend to 0 , as $i \rightarrow \infty$. The points $a_{-1}^{(i)}, \ldots, a_{n}^{(i)}$ define a cell of $L_{i}$, hence the open strip $H_{i}$ bounded.by the lattice planes $E_{i}$ and $E_{i}+a_{n}^{(i)}$ is lattice point free. The same is true a fortiori for the simplex $T_{i}=H_{i} \cap \Omega_{h(i)}$. Now

$$
\operatorname{vol}\left(T_{i}\right)=d_{i}^{n}\left(n !\left|e_{1}^{(i)} e_{2}^{(i)} \ldots e_{n}^{(i)}\right|\right)^{-1} \rightarrow \infty(i+\infty),
$$

since the sequence $d_{i}$ has a positive limit and at least one of the components of $\underline{e}^{(i)}$ tends to zero (note that, for $j=1, \ldots, n$, the 
vectors $\underline{a}_{j}^{(i)}$ tend to a limit $\neq \underline{0}$, and that all components of the unit vectors $\underline{e}^{(i)}$ are non-zero by (a), and remain bounded). It follows that

$$
\alpha_{1}\left(L_{i}\right) \rightarrow \infty \quad(i \rightarrow \infty)
$$

Using successively the relations $(5 a),(3 b)$ and $(4 a)$, we obtain

$$
n ! n^{-n} \alpha_{1}\left(L_{i}\right) \leq \delta_{B}\left(L_{i}\right)=\delta_{B}\left(D_{i} L\right)=\delta_{B}(L) \leq c^{-1} \delta_{M}(L) .
$$

Since this holds for all $i=1,2, \ldots$, we end up with $\delta_{M}(L)=\infty$, as required. This completes the proof of the Theorem.

\section{References}

[1] R.P. Bambah, "Geometry of Numbers, packing and covering and discrete geometry", Math. Student 39 (1971), 117-129.

[2] B.J. Birch, and H.P.F. Swinnerton-Dyer, "On the inhomogeneous minimum of the product of linear forms", Mathematika 3 (1956), 25-39.

[3] B. Bullig-Bergmann, "Ein periodisches Verfahren zur Berechnung eines Systems von Grundeinheiten in den total reellen kubischen Körpern", Abh. Math. Sem. Univ. Homburg 12 (1938), 369-414.

[4] B. Bullig-Bergmann, "Zur Kettenbruchtheorie im Dreidimensionalen", Abh. Math. Sem. Univ. Hamburg 13 (1940), 321-343.

[5] B. Bullig-Bergmann, "Zur Kettenbruchtheorie im n-Dimensionalen", Math. Ann. (1941), 1-31.

[6] B. Bullig-Bergmann, "Periodische Ketten linearex Transformationen", J. Reine Angew. Math. (1953), 108-124.

[7] P.M. Gruber, "Geometry of Numbers", in Contributions to Geometry, (Proceedings of the Geometry Symposium in Siegen 1978, J. T8lke and J.M. Wills, Eds., Birkhauser, Basel. 1979), 186-225.

[8] P.M. Gruber, \& G. Ramharter, "Beitrăge zum Umkehrproblem für den Minkowskischen Linearformensatz", Act. Math. Acad. Sci. Hungar. $39(1-3)(1982), 135-141$.

[9] J. Hammer, Unsolved problems conceming lattice points, (Research Notes in Mathematics 15 Pitman, London, San Francisco, Melbourne, 1977). 
[10] E. Hlawka, "über Gitterpunkte in Parallelepipeden", J. Reine Angew. Math. 187 (1950), 246-252.

[11] C.G. Lekkerkerker, Geometry of Numbers, (Wolters-Noordhoff, Groningen and North-Holland, Amsterdam, London, 1969).

[12] G. Ramharter, "Über ein Problem von Mordell in der Geometrie der Zahlen", Monatsh. Math. 92 (1981), 143-160.

[13] G. Ramharter, "Inhomogeneous and asymetric minima of star sets and the diagonal group", submitted to J. Indian Math. Soc.

[14] C.A. Rogers, "On a theorem of Siegel and Hlawka", Ann. of Math. 53 (1951), 531-540.

[15] D.B. Sawyer, "Lattice points and the diagonal group", J.London Math. Soc. 41 (1966), 466-468.

[16] D.B. Sawyer, "Lattice points in rotated star sets", J. London Math. Soc. 43 (1968), 131-142.

Institut für Analysis,

Techn. Universität Wien,

Wiedner Hauptstrasse 8-10,

A-1040 Vienna,

Austria. 